This article has been published in Annals of Biomedical Engineering, Vol. 44, No. 5, May 2016. The final publication is available from Springer via http://doi.org/10.1007/s10439-015-1462-9.

\title{
Surface and Subsurface Analyses of Metal-on-Polyethylene Total Hip Replacement Retrievals
}

Vicky Vuong ${ }^{1}$, Maria Pettersson ${ }^{2}$, Cecilia Persson ${ }^{2}$, Sune Larsson ${ }^{3}$, Kathryn Grandfield ${ }^{* 1}$, Håkan Engqvist ${ }^{2}$

${ }^{1}$ Department of Materials Science and Engineering, McMaster University, Hamilton, ON, Canada

${ }^{2}$ Division of Applied Materials Science, Department of Engineering Sciences, Uppsala University, Uppsala, Sweden

${ }^{3}$ Department of Surgical Sciences-Orthopaedics, Uppsala University Hospital, Uppsala, Sweden

${ }^{*}$ Corresponding Author: Kathryn Grandfield, kgrandfield@mcmaster.ca, (905) 525-9140 ext. 23573. McMaster University, 1280 Main Street West, Hamilton ON L8S 4L8, Canada. 


\section{Abstract}

Metal-on-polyethylene (MoP) articulations are one of the most reliable implanted hip prostheses. Unfortunately, long-term failure remains an obstacle to the service life. There is a lack of higher resolution research investigating the metallic surface component of MoP hip implants. This study investigates the surface and subsurface features of metallic cobalt chromium molybdenum alloy (CoCrMo) femoral head components from failed MoP retrievals. Unused prostheses were used for comparison to differentiate between wear-induced defects and imperfections incurred during implant manufacturing. The predominant scratch morphology observed on the non-implanted references was shallow and linear, whereas the scratches on the retrievals consisted of largely nonlinear, irregular scratches of varying depth (up to $150 \mathrm{~nm}$ in retrievals and up to $60 \mathrm{~nm}$ in reference samples). Characteristic hard phases were observed on the surface and subsurface material of the cast samples. Across all samples, a 100 to 400 $\mathrm{nm}$ thick nanocrystalline layer was visible in the immediate subsurface microstructure. Although observation of the nanocrystalline layer has been reported in metal-on-metal (MoM) articulations, its presence in MoP retrievals and unimplanted prostheses has not been extensively examined. The results suggest that manufacturing-induced surface and subsurface microstructural features are present in MoP hip prostheses prior to implantation and naturally, these imperfections may influence the in vivo wear processes after implantation.

Key terms: hip implant retrievals, cobalt chromium, metal-on-polymer, transmission electron microscopy, biotribology, electron microscopy 


\section{Introduction}

Despite the high success rates during early years of implantation of bearing couples in use today, implant failure after 10 to 15 years imposes a limit on the effectiveness of total hip arthroplasty (THA) ${ }^{1,2}$. Thorough understanding of the wear processes that occur at the interface of hip prostheses requires the correlation between surface wear and subsurface microstructural changes. Previous studies have reported that the predominant wear mechanisms observed for metal-on-metal (MoM) prostheses include abrasion ${ }^{3,4}$, surface fatigue and tribochemical reactions ${ }^{5,6}$. Other researchers have investigated the presence of a tribological layer thought to serve as a lubricating barrier to prevent direct contact in areas of the self-mating system, and thus minimize the effects of adhesive wear ${ }^{3}$. However, the mechanism of formation and the composition of this layer is still under debate. Since the primary source of carbon in the formation of a carbon-rich layer on the surface of MoM articulations in hip simulator studies originates from the bovine serum lubricant, researchers suggest that the layer is organic and protein-derived ${ }^{6}$. If the tribological layer acts as a lubricant, it may hinder the progression of wear and corrosion and in turn, reduce wear rates. However, low volumetric wear is not necessarily correlated with long-term performance. The morphology, composition, and biological impact of the generated wear debris can play a greater role in contributing to the failure of the prosthesis. Therefore, in vivo biochemical interactions producing wear debris may introduce adverse physiological complications ${ }^{7-}$ 9. Overall, while the characterization of the surface and subsurface features on the metallic component has been explored in MoM hip prostheses ${ }^{10-14}$, it has been lacking in the literature for the metallic surface of metal-on-polyethylene (MoP) hip retrievals, which comprise the most widely implanted bearing combination, internationally ${ }^{15-17}$. 
In difference to the self-mating metallic articulation seen in MoM prostheses, MoP prostheses commonly consist of an ultra-high molecular weight polyethylene (UHMWPE) or highly cross-linked UHMWPE (HXLPE) acetabular cup coupled to a cobalt chromium molybdenum alloy (CoCrMo) femoral head. The knowledge acquired from MoM research can serve as a foundation for understanding some of the mechanisms that may drive the failure of MoP hip prostheses. However, since both systems operate under distinct tribological regimes ${ }^{9,18}$, the results from investigations of MoM interfaces cannot be directly extrapolated to MoP articulations. More recently, much of the research on MoP prostheses has been focused on the modification of the polymer counterpart to reduce the release of polyethylene wear debris. However, the interaction at the articulating surface of MoP prostheses may be insightful to resolving the mechanism of implant failure. Polyethylene debris from the polymer acetabular cup and metallic debris generated from the modular surfaces are the most commonly reported sources for the accumulation of wear debris (the main culprit for debrisinduced osteolysis) in MoP implants ${ }^{19,20}$. Although in a well-functioning MoP implant, wear particles mainly originate from the polymeric articulating component, metallic particles originating from e.g. modular components (as in many MoM implants) can migrate into the articulating space and act as a source of third-body abrasive wear promoting wear on the metallic articulating surface ${ }^{21,22}$. Embedded metal particles in the polyethylene have also been clinically reported in retrievals ${ }^{23}$ and can lead to abrasion onto the complimentary metallic surface ${ }^{24,25}$. In extreme conditions of malfunction or dislocation, the femoral head has been noted to generate debris against the shell of the acetabular cup ${ }^{24}$. Consequently, the corrosion and wear resistance of 
the surface of the metallic femoral head can be influenced by the effects of third-body wear and in turn exacerbate the wear of the polyethylene component as demonstrated in simulated studies ${ }^{26,27}$. Therefore, investigations regarding the wear features of the metallic surface should not be overlooked ${ }^{28,29}$. This study aims to examine the surface wear, tribological layer, and the subsurface microstructure of the metallic femoral head component in MoP THA prostheses retrieved from humans after 2 to 15 years and compare the results with previous studies on MoM articulations. In this study, advanced microscopy techniques including vertical scanning interferometry, scanning electron microscopy, energy dispersive spectroscopy, auger electron spectroscopy, focused ion beam, and transmission electron microscopy were used to characterize features from the metallic component of MoP prostheses.

\section{Materials and Methods}

\subsection{Samples and Sample Preparation}

Three MoP hip femoral heads were retrieved from patients at Uppsala University Hospital, Sweden in 2010. All retrievals were removed for a primary revision surgery due to instability or aseptic loosening and wear. The two reference samples included in this study were an unused wrought CoCrMo alloy femoral head and a low carbon cast CoCrMo puck (manufactured by the same process as femoral heads). Specific sample details and patient data are provided in Table 1. The manufacturer and implant information is unknown for Retrieval Explant 3. Polishing, etching and elemental analysis of the bulk sample revealed the presence of large grain boundaries and an abundance of carbides in the bulk microstructure suggesting that it is a cast CoCrMo alloy. In addition, the lack of carbides in the wrought samples suggest that they are low carbon wrought CoCrMo alloys. 
The retrievals and reference samples were ultrasonically cleaned in detergent solution (UltraClean TopDent, Upplands-Väsby, Sweden) for multiple 15 min cycles to dissolve bone cement, protein, blood residue, and fat. Following this, the samples were ultrasonically cleaned in ethanol. All samples were cut using electrical discharge machining (EDM) in the transverse plane to minimize the size of the samples (approximately $16 \mathrm{~mm}$ diameter by $4 \mathrm{~mm}$ height) to allow for insertion into the auger electron spectroscopy (AES) microscope. This also allowed for stabilization of the sample during tilt angles in the focused ion beam (FIB) microscope. Immediately prior to electron microscopy, samples were rinsed with detergent for $5 \mathrm{~min}$, washed with distilled water, ultrasonically washed in acetone for $5 \mathrm{~min}$, ultrasonically washed in methanol for $5 \mathrm{~min}$ and allowed to air dry. For further analyses, the superior, medial region of the femoral head samples was used to maintain consistency in the investigated areas of interest since in vivo loading conditions are uncertain and inconsistent between samples.

\subsection{Scanning Electron Microscopy}

To characterize surface wear, all samples were investigated using the JEOL JSM-6610LV (JEOL, Japan) tungsten filament scanning electron microscope (SEM). The microscope was operated at accelerating voltages between $2 \mathrm{keV}$ to $15 \mathrm{keV}$ and beam currents of 40 to $60 \mathrm{pA}$. The secondary electron imaging (SEI) mode and backscattered electron (BSE) imaging mode were used simultaneously to analyze surface

topography and elemental compositional contrast. Energy dispersive X-ray spectroscopy (EDS) analysis between $10 \mathrm{keV}$ to $15 \mathrm{keV}$ was also used to perform point identifications, line-scans and elemental mapping of the surface of the samples. 


\subsection{Vertical scanning interferometry}

The surface roughness and appearance of the top and side of the retrievals were evaluated using vertical scanning interferometry (VSI, Wyko NT-1100, Vecco Instruments, USA) with a 10X objective, over an area of $590 \times 450 \mu \mathrm{m}$. With the software Vison 3.44 a spherical shape was removed in order to plot and evaluate surfaces on the femoral heads, and no other filtering of the data was applied.

\subsection{Auger Electron Spectroscopy}

AES on a JEOL JAMP 9500F (JEOL, Japan) field emission auger microprobe was used to perform depth profiling on the surface of the samples to characterize the potential presence of a surface film layer. Depth profiling conditions were at $10 \mathrm{keV}, 50$ $\mathrm{nA}, 0.75 \mathrm{eV}$ step size, 5 sweeps and $100 \mathrm{~ms}$ dwell time. The estimated sputtering rate for a generic CoCrMo alloy was $30 \mathrm{~nm} / \mathrm{min}$ as indicated in the JAMP-9500 Instruction Manual Appendix 9: Ion Beam Etch and Sputtering Rate ${ }^{30}$.

\subsection{Focused Ion Beam}

To isolate specific sites of interest for cross-sectional examination of subsurface microstructure and crystallinity using the transmission electron microscope (TEM), conventional FIB milling procedures were performed using the Zeiss NVision 40 (Carl Zeiss, Germany) FIB SEM (equipped with a Schottky Field Emission Gun) ${ }^{31}$. Regions of interest on references were selected based on uniform scratches that likely result from manufacturing processes. Regions of interest on retrievals were selected based on locations where deeper scratches were present and if possible, the simultaneous presence of what appeared to be a residual tribological layer on the surface. A protective layer of carbon followed by a thin layer of tungsten was deposited over areas 
of interest to prevent ion beam damage from the bulk milling procedure. Trenches were milled around the area of interest using a $30 \mathrm{keV}$ beam of $\mathrm{Ga}^{+}$ions. The ion gun was then used to mill around the lamella to detach it from the bulk sample, the lamella was lifted out by a micromanipulator, attached onto a copper grid and thinned to electron transparency with a $10 \mathrm{keV}$ beam. Two cross-section TEM samples were prepared for Retrievals 2 and 3 and one cross-section TEM sample was examined for all other samples.

\subsection{Transmission Electron Microscopy}

TEM was used to examine the subsurface microstructure and chemical composition of all samples. A Philips CM12 (FEl, The Netherlands) with LaB6 filament (120 keV) was used for preliminary imaging of subsurface microstructure. A JEOL 2010F (JEOL, Japan) field emission TEM/STEM was operated at $200 \mathrm{keV}$ for EDS mapping and bright-field scanning transmission electron microscopy (STEM) of all samples.

\section{Results}

\subsection{Surface Characterization with SEM, VSI and AES}

Scratches were clearly visible on the surface of the reference and retrieved CoCrMo THA femoral heads even at low resolutions in the SEM. The wear patterns on the unused reference femoral heads (Fig. 1A, 1C) consisted of straight, parallel or perpendicular, multidirectional scratches. In comparison, the surface of the retrievals (Fig. 1B, 1D) were more worn and consisted of many more deep, and non-linear scratches, in addition to the linear, parallel and perpendicular scratches seen in the reference samples. Complementary to SEM results, VSI of the tops and sides of the 
femoral heads showed a higher concentration of scratches on the sides of the retrievals compared to the top and the approximate range of scratch depths were up to $150 \mathrm{~nm}$ deep on the retrievals, while the maximum depth on the investigated areas of the reference samples was $60 \mathrm{~nm}$ (Fig. 2).

Hard phases were visible on the surface of the cast samples. Hard phases were easily identified in the SEM where the scratches are noncontinuous. The hard phases were abundantly found on Retrieval 2 (Fig. 1D arrowhead, Fig. 2, Fig. 3A) and Retrieval 3 (Fig. 2, Fig. 3B) and measured approximately $10-50 \mu \mathrm{m}$ in length (SEM) and up to 0.7 $\mu \mathrm{m}$ in height (VSI). The hard phases were easily differentiated in BSE mode as they appeared brighter due to a composition of elements with higher atomic number compared to the bulk material. On Reference 2, phases that resembled carbides and were $5-10 \mu \mathrm{m}$ (SEM) (Fig. 1C arrow) in length and up to $0.1 \mu \mathrm{m}$ in height (VSI) (Fig. 2), were observed on the surface. However, the hard phases on this reference sample had a darker contrast compared to the surrounding bulk material (Fig. 1C arrow). To further characterize the composition of the hard phases, line-scans using SEM EDS were taken over parts of the bulk material and identifiable hard phases (Fig. 3). In Retrieval 2, the line-scan showed a relatively large enrichment of $\mathrm{Mo}$ and $\mathrm{Cr}$, a small enrichment of $\mathrm{Si}$ and $\mathrm{C}$, as well as an obvious depletion of $\mathrm{Co}$ in the hard phase (Fig. 3A, graph). In Retrieval 3, the linescan showed an enrichment in $\mathrm{Mo}$ and $\mathrm{Si}$, slight enrichment of $\mathrm{Cr}$ (to a lesser extent than in Retrieval 2) and nearly no depletion of Co (Fig. 3B, graph). In addition, VSI results showed that the height of hard phases in Retrieval 2 and 3 was larger on the side of the femoral head compared to the top. No carbides or Mo-rich hard 
phases were identified using SEM or VSI in the wrought Reference 1 sample or the wrought Retrieval 1 sample.

In SEI mode, at lower accelerating voltages (e.g. $10 \mathrm{keV}$, Fig. 4A), an organic layer was evident on the surface of retrievals. The surface of Retrieval 3 (Fig. 4A) was covered in a layer of darker and brighter surface components which masked the distribution of Mo-rich hard phases invisible unless imaged at higher accelerating voltages or when imaged in BSE mode (Fig. 4B). This effect was not observed on Reference 2; the hard phases were evident on the surface and no darker or brighter patches were visible. The AES depth profiles (Fig. 4C, 4D, 4E) were taken at the points labelled +1 (Fig. 4C), +2 (Fig. 4D), +3 (Fig. 4E) on the corresponding reference and retrieval samples. The relative abundance of elements in the bulk material of Reference 2 (Fig. 4E) and the brighter spot on Retrieval 2 (Fig. 4F) were relatively similar with the exception of a lower amount of $\mathrm{C}$ and a larger amount of $\mathrm{O}$ on Retrieval 2. The depletion of $\mathrm{O}$ corresponded to a sputtering time $0.33 \mathrm{~s}$, which, using the estimated sputtering depth rate of $30 \mathrm{~nm} / \mathrm{min}{ }^{30}$, corresponded to an O-rich layer that was approximately $10 \mathrm{~nm}$ in depth. In the darker region on the surface of Retrieval 3 , the depth profile showed a larger amount of $C$ that was depleted at around $1 \mathrm{~s}$, which corresponded to a C-rich layer of approximately $30 \mathrm{~nm}$.

Lastly, in the TEM, (Fig. 5E, 5F, 6D), the residual layer was also evident approximately $10 \mathrm{~nm}$ from the surface of Retrieval 3. TEM EDS showed that this layer had a large composition of $\mathrm{C}$ and $\mathrm{O}$ and small amounts (approximately 2-3\%) of $\mathrm{Ca}$ and Si were also detected.

\subsection{Subsurface Microstructure Characterization with TEM}


FIB was used to prepare TEM samples over the cross-section of scratches on the surface of the samples. The scratches on the references were most likely a result of the manufacturing process and the scratches on the retrievals were selected based on irregular nonlinear appearance, indicative of scratches formed as a result of in vivo wear (Fig. 5A, 5D). Figure 5 shows the cross-sectional microstructure of said scratches imaged in the TEM at lower magnifications (Fig. 5B, 5E) and at higher magnifications (Fig. 5C, 5F). A distinct nanocrystalline layer characterized by smaller grains directly under the surface of the retrievals was evident. The layer appeared to dip lower, following the contours formed by the scratches (Fig. 5B, 5F).

Observations of the subsurface microstructure of all other samples using brightfield STEM revealed a nanocrystalline layer at the surface (Fig. 6). The nanocrystalline layers ranged from $100 \mathrm{~nm}$ to $350 \mathrm{~nm}$ and were present subsurface in both the reference samples (Fig. 6A) and the retrievals (Fig. 7B-7D).

Lastly, to identify the chemical composition of the nanocrystalline layer and subsurface bulk material, EDS in the TEM was used to map small areas in each sample. Selecting a representative case, Fig. 7A shows the STEM micrograph of the Retrieval 2 sample where the EDS map was taken and a refined microstructure was visible across the entire subsurface region. The EDS maps (Fig. 7B-7E) show an area corresponding to a Mo-rich hard phase similar to the ones shown in Figure 2D and Figure 5A. Consistent with the results obtained from the line-scan using EDS in the SEM, the hard phase was rich in $\mathrm{Mo}$ and $\mathrm{Cr}$ and depleted in Co. The refined microstructure appeared continuous between the hard phase and the nanocrystalline layer (Fig. 8B). 


\section{Discussion}

\subsection{Surface wear}

The abundant scratches on the reference samples were likely formed by mechanically-induced wear modes during the proprietary polishing process used during manufacturing. Therefore, the surface wear on the retrieved samples can be attributed to both the manufacturing process and in vivo wear. The shallower, linear, parallel or perpendicular scratches present on both reference samples and retrieved implant samples are suggestive of single-cycle deformation abrasive wear modes ${ }^{32,33}$. On the other hand, the deeper, nonlinear individual scratches may be suggestive of third body abrasive wear ${ }^{28}$. More abundant, complex wear scars and cracks may be a result of repeated-cycle deformation mechanisms ${ }^{32,33}$. The wear patterns observed on the reference samples prior to implantation may have an influence on the wear modes and tribochemical reactions in the mechanical and chemical environment of the in vivo joint. Preexisting scratches and scratches produced in vivo have an effect on the shape, amount and size of wear debris generated ${ }^{33,34}$. The wear and the surface roughness of the retrievals showed a large variation, which is dependent on the positioning in vivo. On the top (superior, medial region) the wear was less adverse than laterally, in a region close to the equator, where the contact pressure and ingress of third body wear particles were most likely higher compared to the pole of the metallic head. On the sides the scratches were deeper and the hard phases (when apparent) were raised slightly above the bulk surface. Presumably, the greater exposure of hard phases on the cast samples, especially at high wear zones, will increase abrasive wear on the complimentary polyethylene component, potentially increasing the generation of wear debris $^{35,36}$. 


\subsection{Carbides and hard phases}

The identification and characterization of hard phases on the surface of the samples are consistent with previous findings in MoM CoCrMo prostheses ${ }^{37,38}$. From examination of the surface of the samples, clusters of hard phases were identified on the cast Reference 2 (Fig. 2C) but not on the surface of wrought Reference 1 and these hard phases differed from hard phases observed on the other retrieved implant samples. The hard phases on Reference 2 most closely resemble the Co-rich $\mathrm{M}_{23} \mathrm{C}_{6}$ and $\mathrm{MC}_{6}$ carbides reported by Bettini et al. ${ }^{38}$. The dissolution of interdendritic carbides and the precipitation of lamellar carbide eutectic phases at grain boundaries are formed by high temperature sintering cycles during the metallurgical modifications of the alloy 39. The most commonly observed hard phases may contain varying compositions of the alloying elements $(M=C o, C r, M o)$ in two crystallographic structures $M_{23} C_{6}$ and $M_{6} C$, found to coexist in large clusters ${ }^{37-39}$. Previous studies suggest that carbides within the grains increase the effective strength of the CoCrMo, but when found at grain boundaries, the carbides tend to embrittle the material ${ }^{37}$. In the MoP tribosystem, due to their high modulus, hard phases likely serve as contact asperities during loading conditions in vivo possibly leading to abrasion of the contacting polymer surface. Wimmer et al. has shown that under high local contact stresses, the hard phases can be removed from the bulk alloy inducing surface fatigue by indentations and third body abrasion ${ }^{3}$. The presence of hard phases and carbon content also has an effect on the passivity of the alloy. The preferential metallic dissolution at hard phase boundaries depletes the metal matrix in $\mathrm{Cr}$, thus hindering the availability of $\mathrm{Cr}$ to form an oxide film

38. Therefore, lowering carbon content (up to $0.35 \%$ carbon can be incorporated into the 
CoCrMo) and increasing the amount of chromium available can generate a more homogenous surface with increased corrosion resistance ${ }^{39}$.

A mixed-phase hard constituent was also found throughout the bulk of the cast retrieval samples (Retrieval 2, Retrieval 3, Fig. 3) and congruent with the Mo-rich $\mathrm{M}_{23} \mathrm{C}_{6}$ carbides as reported in previous MoM studies ${ }^{37,40}$. From high magnifications in the SEM and the varying peak intensities on the EDS line-scans, it was evident that the composition within the hard phases was not homogenous. The hard phases on the retrieval samples were larger in size and had a higher enrichment of Mo in comparison to the hard phases found on cast Reference 1. Furthermore, the elemental profile of hard phases in Retrieval 2 is different from those found in Retrieval 3 - the Retrieval 3 hard phase has a more notable enrichment of Si and no depletion of Co. At higher magnifications, the BSE SEM micrographs show suspended particulates of varying compositional contrasts in the hard phases on the retrievals (Fig. 3). Additionally, the EDS line-scans through the hard phases show peaks of varying relative intensities demonstrating the heterogeneous nature of the hard phases. For example, in Fig. 3B, the spikes in Mo on the EDS line-scan correspond to particles on the SEM micrograph that are brighter, suggesting a higher Mo content in those particles. The nanograin heterogeneity of the hard phase is also evident in the TEM cross-section in Fig. 7. The TEM EDS map shows grains with an abundance in Co, $\mathrm{Cr}$ or Mo (Fig. 7C, 7D, 7E). In the characterization of hard phases found in cast and wrought alloys, Liao et al. found that these mixed-phase hard constituents were found mainly in cast alloys $\left(M_{23} C_{6}\right.$-type and FCC phases) and carbides within the wrought alloys were largely homogenous $\left(\mathrm{M}_{23} \mathrm{C}_{6} \text { single phase }\right)^{37}$. The heterogeneity supports that Retrieval 2 and 3 were formed 
using the casting method. The homogeneity and scarcity of hard phases within wrought alloys may be attributed to the repeated heating and deformation cycles after casting ${ }^{37}$.

In vitro simulator tests have shown that detachment of nanograins can result from the sliding over protruding mixed hard phases leaving pits in the bulk material ${ }^{37}$. Furthermore, cracks propagated through the nanograins of the mixed hard phase after five million cycles while, contrarily, the single phase carbides were still intact ${ }^{41}$. Hard phases, including the mixed hard phases, may be contact asperities that encourage abrasive wear, adhesive wear and surface fatigue on the metallic surface as well as on the UHMWPE counterpart. In addition to the scratches, hard phases may also influence the size, shape and amount of polyethylene wear debris generated.

\subsection{Tribological layer and oxide layer}

Compared to the reference sample (Fig. 4C), the brighter layer in the SEM contains an enrichment of oxygen that suggests the presence of a thin oxide layer (containing chromium oxide (III)) which forms spontaneously as CoCrMo alloys are exposed to air ${ }^{42}$. The depth profile shows the depletion of oxygen at approximately 10 $\mathrm{nm}$ which roughly corresponds to the $8-20 \mathrm{~nm}$ thickness as reported in other MoM tribochemical reaction studies ${ }^{6,9}$.

The AES depth profile of the darker layer in SEM revealed an abundance of carbon and a depletion of the other analyzed elements (Fig. 4E). The approximate thickness of the carbon-rich layer was $30 \mathrm{~nm}$. Milošev and Remškar reported the presence of a profuse solid deposit containing $\mathrm{C}, \mathrm{N}$, and $\mathrm{O}$ that was $1 \mu \mathrm{m}$ thick and a thinner mixed deposit layer containing metal oxides, organic-related components and hydroxides, based on MoM retrievals ${ }^{6}$. The authors of this study suggest that the 
abundance of carbon is organic in nature and is a result of tribochemical reactions that generate compact and partly segmented carbon-containing layers of denatured proteins. Other studies, found that the tribolayers are nanocrystalline in structure and by the process of "mechanical mixing", the organic material from the synovial fluid becomes incorporated into the top $50-200 \mathrm{~nm}$ of the metallic surface ${ }^{3},{ }^{5,9,43-45}$. The carbon-rich tribological layer has been suggested to function as a solid lubricant in the MoM tribological system, reducing direct metal on metal contact and effectively improving wear performance ${ }^{46}$. More recently, Liao et al. proposed the presence of graphitic carbon in the tribological layer which may function more like the lubricant in a combustion engine ${ }^{8}$. In this study, the layer found on Retrieval 3 is comparably thinner than the layers previously reported for MoM retrievals and does not appear to contain similar graphitic carbon in the surface films. However, TEM EDS of the layer on Retrieval 3 revealed a composition of $\mathrm{C}, \mathrm{O}, \mathrm{Si}$ and $\mathrm{Ca}$, supportive that this layer may have an organic component. In the TEM, a thick carbon-rich layer to the same extent has also not been observed in the retrieved samples. Although the TEM samples were protected by a layer of sputtered $\mathrm{C}$, this was under the assumption that a tribological layer containing compacted organic material or a nanocrystalline structure may be differentiated. In contrast to articulations within the MoM joint interface, the appearance of the $60 \mathrm{~nm}$ thick carbon layer observed by AES depth profiling for retrieved MoP joints could be attributed to many sources including: the polymeric acetabular component of the bearing couple, in vivo organic compounds, and exposure to air on the surface of Reference 1 (not shown) and Reference 2 (Fig. 4C), AES depth profiling showed high initial levels of carbon, approximately $10-20 \mathrm{~nm}$ in thickness. Therefore despite the 
slight difference in depth profiles between the reference and the retrieved surfaces, the presence of a carbon-rich nanocrystalline or mechanically-mixed tribological layer (of the same size as MoM studies) on the retrieved samples cannot be ascertained.

\subsection{Subsurface Microstructure}

In 2005, Büscher et al. reported a nanocrystalline layer (500 nm in thickness) in the subsurface microstructure of MoM retrievals within the retrieved acetabular cups [10]. Although mechanical polishing was considered as a possible mechanism of the formation of the nanocrystalline layer, it was suggested that plastic deformation at that stage was insufficient to induce nanocrystal formation. The conclusions drawn from the study were that plastic deformation during the wear process leads to mechanical milling and dynamic recrystallization at the immediate subsurface layer. Furthermore, surface fatigue (and not abrasion) leads to the generation of globular wear debris torn off from the nanocrystalline layer. Moreover, it is suggested that a high number of grain boundaries and defects at the nanocrystalline subsurface layer should increase the mechanical stability and tribological properties of the material [9]. In line with the possibility that a nanocrystallline layer is formed due to polishing during manufacturing, Pourzal et al. have shown the presence of a nanocrystalline layer in the non-contacting zone, however the surface integrity of the sample was not well preserved and only one sample area was demonstrated ${ }^{45}$. In this study, TEM revealed that the nanocrystalline layer was present on both the reference and retrieved samples (Fig. 7). The preparation of TEM samples from the retrievals involved identifying scratches that may not have been present as a result of the manufacturing process (i.e. curved, nonlinear scratches characteristic of in vivo wear). The cross-section of a scratch can provide important 
information, such as the microstructure of the material removed during the creation of wear debris as a result of scratch formation. The layer thicknesses ranged from 100 to $350 \mathrm{~nm}$. However, it should be noted that the lower end of this range corresponds with a retrieved sample (Retrieval 1) and not Reference 1. This suggest that the nanocrystalline layer is generated by the mechanical polishing process during manufacturing of the components and is not entirely due to dynamic recrystallization induced by in vivo wear. However, the creation of the nanocrystalline layer as a result of wear should not be dismissed. Studies on MoM articulations as well as alumina articulations have shown run-in wear that removes up to $250 \mathrm{~nm}$ from the surface which may account for the variable thickness of the nanocrystalline layer in the retrieval samples. Nonetheless, the extent of run-in wear on the metallic component in MoP bearing couples is significantly less in comparison to MoM bearings ${ }^{47-51}$. Observing the cross section of scratches in Fig. 6, the nanocrystalline layer under the area of a scratch appears to follow the contours of the scratch. The thickness of the nanocrystalline layer under the scratches does not appear to be significantly reduced as one would expect if an abrasive scratch were to remove the material at the surface. It appears that a nanocrystalline zone can be generated in the secondary shear zone from an abrading particle, however other studies have shown little to no nanocrystalline zone formation beneath scratches. Further high resolution TEM of the subsurface microstructure on a larger number of retrievals is required to elucidate a deeper understanding of the formation of and wear mechanism caused by the nanocrystalline layer.

In conclusion, many of the results from this study are in agreement with features previously examined in MoM studies. The comparison of retrieved CoCrMo femoral 
heads with unused reference samples shows that there are pre-existing imperfections inherent to the manufacturing process in addition to subsurface microstructural changes associated with in vivo loading conditions. Prior to implantation, a typical unused hip prosthesis may contain regular, linear scratches that may have resulted from the last polishing step of the manufacturing process. Further, at a subsurface microstructural level, the unused prosthesis may contain hard phases and a nanocrystalline layer, all of which may contribute to the wear performance of the MoP prosthesis. Future investigations on a larger sample size, in combination with the complimentary UHMWPE acetabular cup may reveal additional information about the wear mechanisms and tribological interactions between the MoP articulating surfaces.

\section{Acknowledgements}

Funding from the European Union's Seventh Framework Programme (FP7/20072013), under grant agreement no. GA-310477 is gratefully acknowledged.

Funding from the Natural Sciences and Engineering Research Council of Canada (NSERC) Discovery Grant Program and the European Union's Seventh Framework Programme (FP7/2007-2013), under grant agreement no. GA-310477 is gratefully acknowledged. Electron microscopy was performed at the Canadian Centre for Electron Microscopy at McMaster University, a facility supported by NSERC and other government agencies 


\section{References}

1. Kurtz SM, Ong KL, Lau E, Bozic KJ. Impact of the Economic Downturn on Total Joint Replacement Demand in the United States Updated Projections to 2021. J Bone Joint Surg Am. 2014;96(8):624-630.

2. Jenkins PJ, Clement ND, Hamilton DF, Gaston P, Patton JT, Howie CR. Predicting the cost-effectiveness of total hip and knee replacement: A health economic analysis. J Bone Jt Surg - Ser B. 2013;95 B(1):115-121. doi:10.1302/0301-620X.95B1.29835.

3. Wimmer MA, Loos J, Nassutt R, Heitkemper M, Fischer A. The acting wear mechanisms on metal-on-metal hip joint bearings: in vitro results. Wear. 2001;250(1-12):129-139. doi:10.1016/S0043-1648(01)00654-8.

4. Büscher R, Fischer A. Metallurgical Aspects of Sliding Wear of fcc Materials for Medical Applications. Materwiss Werksttech. 2003;34(1011):966-975. doi:10.1002/mawe.200300680.

5. Wimmer MA, Sprecher C, Hauert R, Täger G, Fischer A. Tribochemical reaction on metal-on-metal hip joint bearings. Wear. 2003;255(7-12):1007-1014. doi:10.1016/S0043-1648(03)00127-3.

6. Milosev I, Remskar M. In vivo production of nanosized metal wear debris formed by tribochemical reaction as confirmed by high-resolution TEM and XPS analyses. J Biomed Mater Res A. 2009;91(4):1100-1110.

doi:10.1002/jbm.a.32301.

7. Mathew MT, Nagelli C, Pourzal R, et al. Tribolayer formation in a metal-on-metal (MoM) hip joint: an electrochemical investigation. J Mech Behav Biomed Mater. 2014;29:199-212. doi:10.1016/j.jmbbm.2013.08.018.

8. Liao Y, Pourzal R, Wimmer MA, Jacobs JJ, Fischer A, Marks LD. Graphitic tribological layers in metal-on-metal hip replacements. Science. 2011;334(6063):1687-1690. doi:10.1126/science.1213902.

9. Wimmer MA, Fischer A, Büscher R, et al. Wear mechanisms in metal-on-metal bearings: the importance of tribochemical reaction layers. $J$ Orthop Res. 2010;28(4):436-443. doi:10.1002/jor.21020.

10. Büscher R, Täger G, Dudzinski W, Gleising B, Wimmer M a, Fischer a. Subsurface microstructure of metal-on-metal hip joints and its relationship to wear particle generation. J Biomed Mater Res B Appl Biomater. 2005;72(1):206-214. doi:10.1002/jbm.b.30132. 
11. Pourzal R, Morlock M, Ma W, Fischer A. Are Micro-Structural Changes in MoM Hip Resurfacings Comparable to Total Hip Arthroplasties ? Poster No . 2360 • 55th Annual Meeting of the Orthopaedic Research Society. 2006;(2360):2360.

12. Fischer A. Subsurface microstructural alterations during sliding wear of biomedical metals. Modelling and experimental results. Comput Mater Sci. 2009;46(3):586-590. doi:10.1016/j.commatsci.2009.01.016.

13. Pourzal R, Theissmann R, Morlock M, Fischer a. Micro-structural alterations within different areas of articulating surfaces of a metal-on-metal hip resurfacing system. Wear. 2009;267(5-8):689-694. doi:10.1016/j.wear.2009.01.012.

14. Brostow W, Gorman BP, Olea-Mejia O. Focused ion beam milling and scanning electron microscopy characterization of polymer+metal hybrids. Mater Lett. 2007;61(6):1333-1336. doi:10.1016/j.matlet.2006.07.026.

15. Wishart N, Beaumont R, Young E, Mccormack V, Swanson M. National Joint Registry: 11th Annual Report. 2014;(December 2013).

16. Rogmark C, Rolfson O. Swedish Hip Arthroplasty Register.; 2012.

17. Information $\mathrm{Cl}$ for $\mathrm{H}$. Hip and Knee Replacements in Canada : Canadian Joint Replacement Registry 2013 Annual Report. 2013.

18. Affatato S, Zavalloni M, Taddei P, Di Foggia M, Fagnano C, Viceconti M. Comparative study on the wear behaviour of different conventional and crosslinked polyethylenes for total hip replacement. Tribol Int. 2008;41(8):813-822. doi:10.1016/j.triboint.2008.02.006.

19. Zhu YH, Chiu KY, Tang WM. Review Article: Polyethylene wear and osteolysis in total hip arthroplasty. J Orthop Surg (Hong Kong). 2001;9(1):91-99. http://www.ncbi.nlm.nih.gov/pubmed/12468851.

20. Tan SC. Effect of Taper Design on Trunnionosis in Metal on Polyethylene Total Hip Arthroplasty . Citation of this paper : 2015.

21. Delaunay C, Petit I, Learmonth ID, Oger P, Vendittoli PA. Metal-on-metal bearings total hip arthroplasty: The cobalt and chromium ions release concern. Orthop Traumatol Surg Res. 2010;96:894-904. doi:10.1016/j.otsr.2010.05.008.

22. Klapperich C, Graham J, Pruitt L, Ries MD. Failure of a metal-on-metal total hip arthroplasty from progressive osteolysis. J Arthroplasty. 1999;14(7):877-881. doi:10.1016/S0883-5403(99)90042-6. 
23. Bloebaum RD, Zou L, Bachus KN, Shea KG, Hofmann AA, Dunn HK. Analysis of particles in acetabular components from patients with osteolysis. Clin Orthop Relat Res. 1997;(338):109-118.

24. Cipriano CA, Issack PS, Beksac B, Della Valle AG, Sculco TP, Salvati EA. Metallosis after metal-on-polyethylene total hip arthroplasty. Am J Orthop (Belle Mead NJ). 2008;37:E18-E25.

25. Heiner AD, Galvin AL, Fisher J, Callaghan JJ, Brown TD. Scratching Vulnerability of Conventional vs Highly Cross-Linked Polyethylene Liners Because of Large Embedded Third-Body Particles. J Arthroplasty. 2012;27(5):742-749. doi:10.1016/j.arth.2011.10.002.

26. Minakawa H, Stone MH, Wroblewski BM, Lancaster JG, Ingham E, Fisher J. Quantification of third-body damage and its effect on UHMWPE wear with different types of femoral head. J Bone Joint Surg Br. 1998;80(5):894-899. doi:10.1302/0301-620X.80B5.8675.

27. Shen F-W, McKellop H. Surface-gradient cross-linked polyethylene acetabular cups: oxidation resistance and wear against smooth and rough femoral balls. Clin Orthop Relat Res. 2005;(430):80-88. doi:00003086-200501000-00010 [pii].

28. Topolovec M, Cör A, Milošev I. Metal-on-metal vs. metal-on-polyethylene total hip arthroplasty tribological evaluation of retrieved components and periprosthetic tissue. J Mech Behav Biomed Mater. 2014;34:243-252.

doi:10.1016/j.jmbbm.2014.02.018.

29. Lindgren JU, Brismar BH, Wikstrom AC. Adverse reaction to metal release from a modular metal-on-polyethylene hip prosthesis. J Bone Jt Surg - Br Vol. 2011;93B:1427-1430. doi:10.1302/0301-620X.93B10.27645.

30. JAMP-9500F Instruction manual.

31. Zeng P, Rainforth WM, Inkson BJ, Stewart TD. Transmission electron microscopy analysis of worn alumina hip replacement prostheses. Acta Mater. 2012;60:20612072. doi:10.1016/j.actamat.2012.01.009.

32. Kato K. Classification of wear mechanisms/models. Proc Inst Mech Eng Part J J Eng Tribol. 2002;216:349-355. doi:10.1243/135065002762355280.

33. Briscoe B. Tribology - Friction and wear of engineering materials. Tribol Int. 1992;25:357. doi:10.1016/0301-679X(92)90040-T.

34. Patten EW, Beitel E, Swan A, et al. Classification of Scratches on Retrieved Cobalt Chrome Humeral Heads Using 3D Profilometry. 58th Annu Meet Orthop Res Soc. 2012;38(1210):Poster No. 1210. 
35. Buford a., Goswami T. Review of wear mechanisms in hip implants: Paper I General. Mater Des. 2004;25(5):385-393. doi:10.1016/j.matdes.2003.11.010.

36. Sun D, Wharton J a., Wood RJK, Ma L, Rainforth WM. Microabrasion-corrosion of cast CoCrMo alloy in simulated body fluids. Tribol Int. 2009;42(1):99-110. doi:10.1016/j.triboint.2008.05.005.

37. Liao Y, Pourzal R, Stemmer P, et al. New insights into hard phases of CoCrMo metal-on-metal hip replacements. J Mech Behav Biomed Mater. 2012;12:39-49. doi:10.1016/j.jmbbm.2012.03.013.

38. Bettini E, Eriksson T, Boström M, Leygraf C, Pan J. Influence of metal carbides on dissolution behavior of biomedical CoCrMo alloy: SEM, TEM and AFM studies. Electrochim Acta. 2011;56(25):9413-9419. doi:10.1016/j.electacta.2011.08.028.

39. Valero-Vidal C, Casabán-Julián L, Herraiz-Cardona I, Igual-Muñoz a. Influence of carbides and microstructure of CoCrMo alloys on their metallic dissolution resistance. Mater Sci Eng C Mater Biol Appl. 2013;33(8):4667-4676. doi:10.1016/j.msec.2013.07.041.

40. Stemmer P, Pourzal R, Liao Y, et al. Metal-On-Metal Total Hip Replacement Devices. Kurtz SM, Greenwald AS, Mihalko WH, Lemons JE, eds. 2013:1-17. doi:10.1520/STP1560-EB.

41. Catelas I, Bobyn JD, Medley JB, Krygier JJ, Zukor DJ, Huk OL. Size, shape, and composition of wear particles from metal-metal hip simulator testing: effects of alloy and number of loading cycles. J Biomed Mater Res A. 2003;67(1):312-327. doi:10.1002/jbm.a.10088.

42. Milošev I, Strehblow HH. The composition of the surface passive film formed on CoCrMo alloy in simulated physiological solution. Electrochim Acta. 2003;48:2767-2774. doi:10.1016/S0013-4686(03)00396-7.

43. Wimmer MA, Mathew MT, Laurent MP, et al. Tribochemical Reactions in Metalon-Metal Hip Joints Influence Wear and Corrosion. Kurtz SM, Greenwald AS, Mihalko WH, Lemons JE, eds. Met Total Hip Replace Devices. 2013:1-18. doi:10.1520/STP1560-EB.

44. Pourzal R, Catelas I, Theissmann R, Kaddick C, Fischer A. Characterization of wear particles generated from CoCrMo alloy under sliding wear conditions. Wear. 2011;271(9-10):1658-1666. doi:10.1016/j.wear.2010.12.045.

45. Pourzal R, Theissmann R, Williams S, Gleising B, Fisher J, Fischer A. Subsurface changes of a MoM hip implant below different contact zones. J Mech Behav Biomed Mater. 2009;2(2):186-191. doi:10.1016/j.jmbbm.2008.08.002. 
46. Goldsmith AA, Dowson D, Isaac GH, Lancaster JG. A comparative joint simulator study of the wear of metal-on-metal and alternative material combinations in hip replacements. Proc Inst Mech Eng H. 2000;214:39-47. doi:124.

47. Jacobs J, Shanbhag a, Glant T, Black J, Galante J. Wear Debris in Total Joint Replacements. J Am Acad Orthop Surg. 1994;2:212-220. http://www.ncbi.nlm.nih.gov/pubmed/10709011.

48. Bhatt H, Goswami T. Implant wear mechanisms--basic approach. Biomed Mater. 2008;3(4):042001. doi:10.1088/1748-6041/3/4/042001.

49. Anissian HL, Stark A, Good V, Dahlstrand H, Clarke IC. The wear pattern in metal-on-metal hip prostheses. J Biomed Mater Res. 2001;58(6):673-678. doi:10.1002/jbm.1068.

50. Clarke IC, Good V, Williams $P$, et al. Ultra-low wear rates for rigid-on-rigid bearings in total hip replacements. Proc Inst Mech Eng H. 2000;214(4):331-347. doi:10.1243/0954411001535381.

51. Alhassan S, Goswami T. Wear rate model for UHMWPE in total joint applications. Wear. 2008;265(1-2):8-13. doi:10.1016/j.wear.2007.08.017. 


\section{Tables and Figure Legends}

Table 1. Detailed information on samples ${ }^{1}$

\begin{tabular}{|c|c|c|c|c|c|c|c|c|c|}
\hline \multicolumn{5}{|c|}{ Implants } & \multicolumn{5}{|c|}{ Patient } \\
\hline Sample & $\begin{array}{l}\text { Size } \\
(\mathrm{mm})\end{array}$ & Material & $\begin{array}{l}\text { Manufacture } \\
\text { r/Model }\end{array}$ & $\begin{array}{l}\text { Duration } \\
\text { in vivo } \\
\text { (years) }\end{array}$ & $\begin{array}{l}\text { Se } \\
x\end{array}$ & $\begin{array}{l}\text { Weight } \\
\text { (kg) }\end{array}$ & $\begin{array}{l}\text { Heigh } \\
t(\mathrm{~cm})\end{array}$ & $\begin{array}{c}\text { Age at } \\
\text { insertio } \\
n\end{array}$ & $\begin{array}{l}\text { Reason } \\
\text { for } \\
\text { Removal }\end{array}$ \\
\hline $\begin{array}{l}\text { Retrieval } \\
\text { Explant } 1\end{array}$ & 28 & $\begin{array}{l}\text { Wrought } \\
\text { CoCrMo } \\
\text { (ISO 5832- } \\
12 ; \text { ASTM } \\
1537 \text { ) }\end{array}$ & Link/Lubinus & 2 & $\mathrm{~F}$ & 69 & 163 & 78 & Instability \\
\hline $\begin{array}{l}\text { Retrieval } \\
\text { Explant } 2\end{array}$ & 28 & $\begin{array}{l}\text { Cast } \\
\text { CoCrMo } \\
\text { (ISO5832-4) }\end{array}$ & Protek/Cone & 15 & $\mathrm{~F}$ & 50 & 160 & 41 & $\begin{array}{l}\text { Aseptic } \\
\text { loosening } \\
\text { /wear }\end{array}$ \\
\hline $\begin{array}{l}\text { Retrieval } \\
\text { Explant } 3\end{array}$ & 28 & $\begin{array}{l}\text { Cast } \\
\text { CoCrMo }\end{array}$ & Unknown & 15 & $F$ & 62 & 156 & 69 & $\begin{array}{l}\text { Aseptic } \\
\text { loosening } \\
\text { /wear }\end{array}$ \\
\hline $\begin{array}{l}\text { Reference } \\
1\end{array}$ & 28 & $\begin{array}{l}\text { Wrought } \\
\text { CoCrMo } \\
\text { (ISO5832- } \\
12 \text { ) }\end{array}$ & Zimmer & - & - & - & - & & - \\
\hline $\begin{array}{l}\text { Reference } \\
2 \\
\text { (Puck) }\end{array}$ & $\begin{array}{l}D=30 \\
H=10\end{array}$ & $\begin{array}{l}\text { Cast } \\
\text { CoCrMo } \\
\text { (ASTM F- } \\
75 \text { ) }\end{array}$ & Sandvik & - & - & - & - & & - \\
\hline
\end{tabular}

\footnotetext{
${ }^{1}$ The manufacturer for Retrieval Explant 3 is unknown but the explant is assumed to be cast based on characterization of microstructure, composition and the abundance of carbides.
} 
Figure 1. SEM micrographs of the surfaces of the references and retrievals. The surfaces of wrought Reference 1 (A) and cast Reference 2 (C) have many straight, uniform, parallel/perpendicular scratches. There is also an abundance of hard phases $(5-10 \mu \mathrm{m})$ distributed on the surface of Reference 2 (C, arrow). The surfaces of the retrievals $(B$ and $D)$ have an abundance of more irregular scratches in addition to the straight parallel scratches. A Mo-rich hard phase (30 $\mu \mathrm{m}$ in length) is visible in the micrograph of Retrieval 2 (D, arrowhead).

Figure 2. Depth of scratches and size of hard phases. VSI (590 X $450 \mu \mathrm{m})$ was used to visualize the depth of scratches as well as the height and size of hard phases.

Figure 3. Molybdenum-rich hard phases on the surface of cast retrievals. EDS line-scans (graphs to the right of the corresponding backscattered SEM micrographs) are taken over the black line through a hard phase, as shown in the SEM micrographs (A, B). The hard phase on Explant 2 (A) has an abundance of $\mathrm{Mo}, \mathrm{Cr}$ and a slight increase Si and C compared to the bulk metal. The hard phase on Explant 3 is highly abundant in $\mathrm{Mo}$ and $\mathrm{Si}$, but shows only a slight increase in $\mathrm{Cr}$, and very little depletion of Co.

Figure 4. SEM and AES depth profiles of oxide layer and tribolayer. SEM micrographs of Explant $3(A, B)$, where the more surface sensitive SEl image (A) shows an evident surface layer of brighter and darker areas than when viewed in BSE (B). The estimated sputtering rate of CoCrMo is approximately $30 \mathrm{~nm} / \mathrm{s}$. The depth profile of the cast Reference 2 bulk material is shown in $(C,+1)$; the depth profile of Explant 2 at a bright spot is shown in ( $D,+2)$; and the depth profile of Explant 3 at a dark spot is shown in $(E,+3)$. Explant $2(D,+2)$ shows higher amounts of oxygen compared to the 
reference, approximately $10 \mathrm{~nm}$ in depth. Explant 3 shows slightly more carbon compared to the reference, approximately $30 \mathrm{~nm}$ in depth.

Figure 5. TEM micrographs taken over scratches on the explant surface. SEM micrographs of scratched regions selected for TEM sample preparation using FIB (A, D). TEM micrographs of the cross-section of scratches are shown at lower magnifications $(B, E)$ and at higher magnifications $(C, F)$ showing both nanocrystalline surface layers, and divots corresponding to removed material in the scratch.

Figure 6. STEM BF micrographs of surface nanocrystalline layer. A distinct nanocrystalline microstructure is visible at the surface of all explant and reference samples $(A-E)$. The arrows delineate the height of the layers which range from approximately $100-350 \mathrm{~nm}$.

Figure 7. EDS map in TEM of the Mo,Cr-rich hard phase. Transmission electron micrograph of Explant 2 (A) that shows refined subsurface nanocrystalline layer. Elemental maps of $\mathrm{Co}(\mathrm{C}), \mathrm{Cr}(\mathrm{D}), \mathrm{Mo}(\mathrm{E})$ were taken over a small area $(\mathrm{B})$. The elemental maps show an abundance of $\mathrm{Mo}$ and $\mathrm{Cr}$ and a depletion of $\mathrm{Co}$ in an area that corresponds to the Mo, Cr-rich phase in the sample. The Mo, Cr-rich phase has a refined structure that continues across the subsurface layer of the sample corresponding to the nanocrystalline layer. 


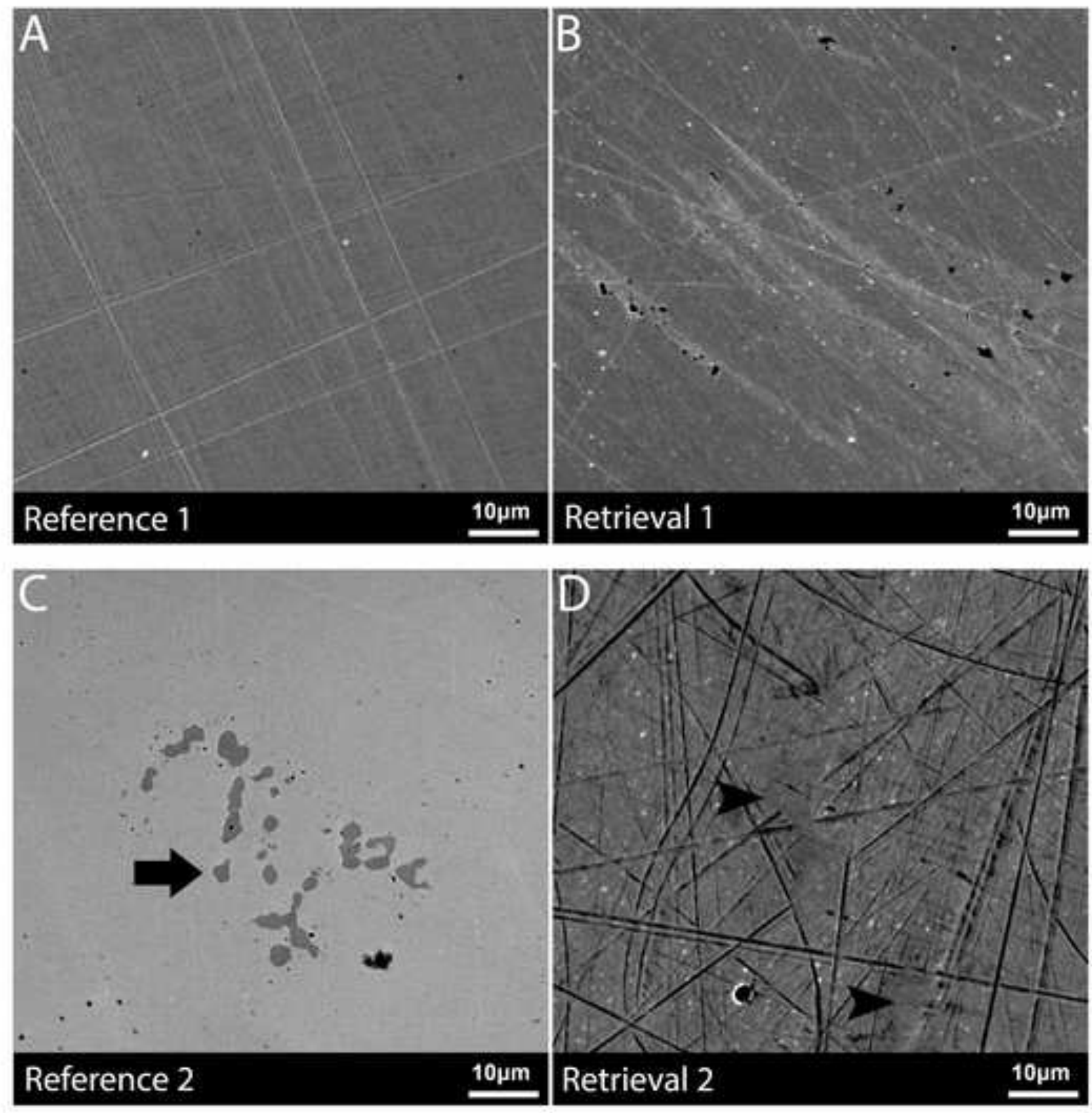

Figure 1 

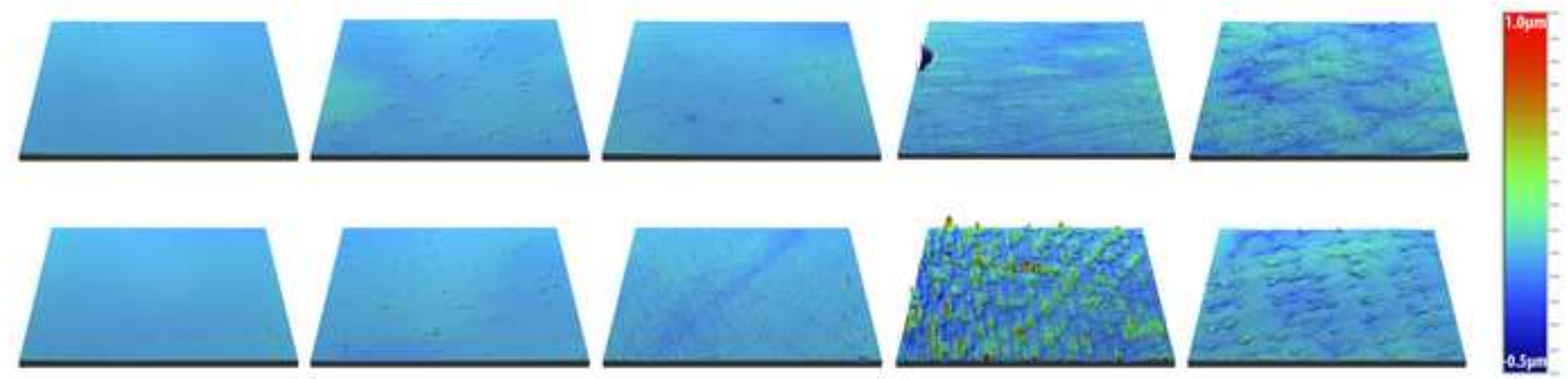

Retrieval 2

Retrieval 3

1590x450um

Figure 2 

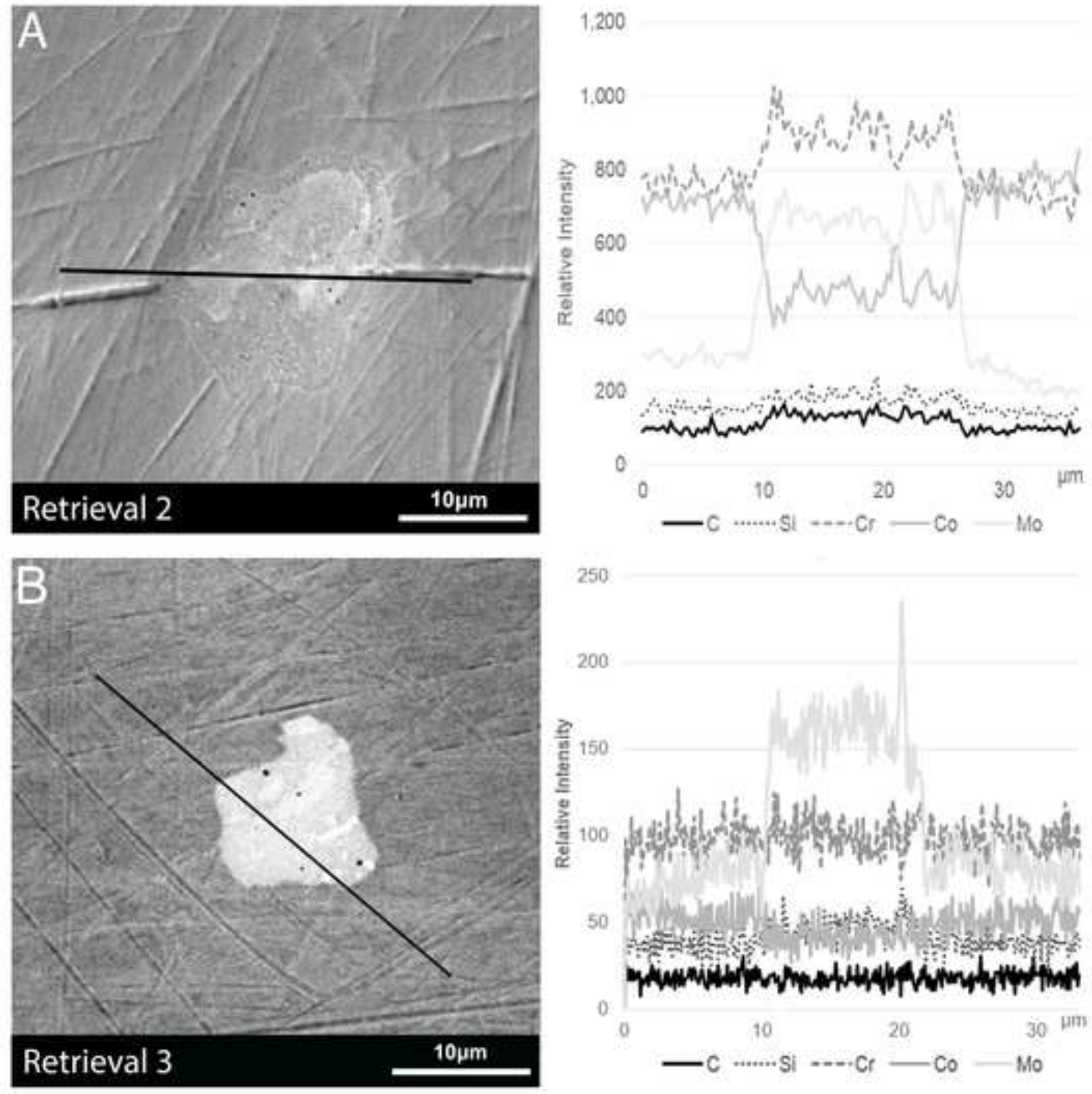

Figure 3 

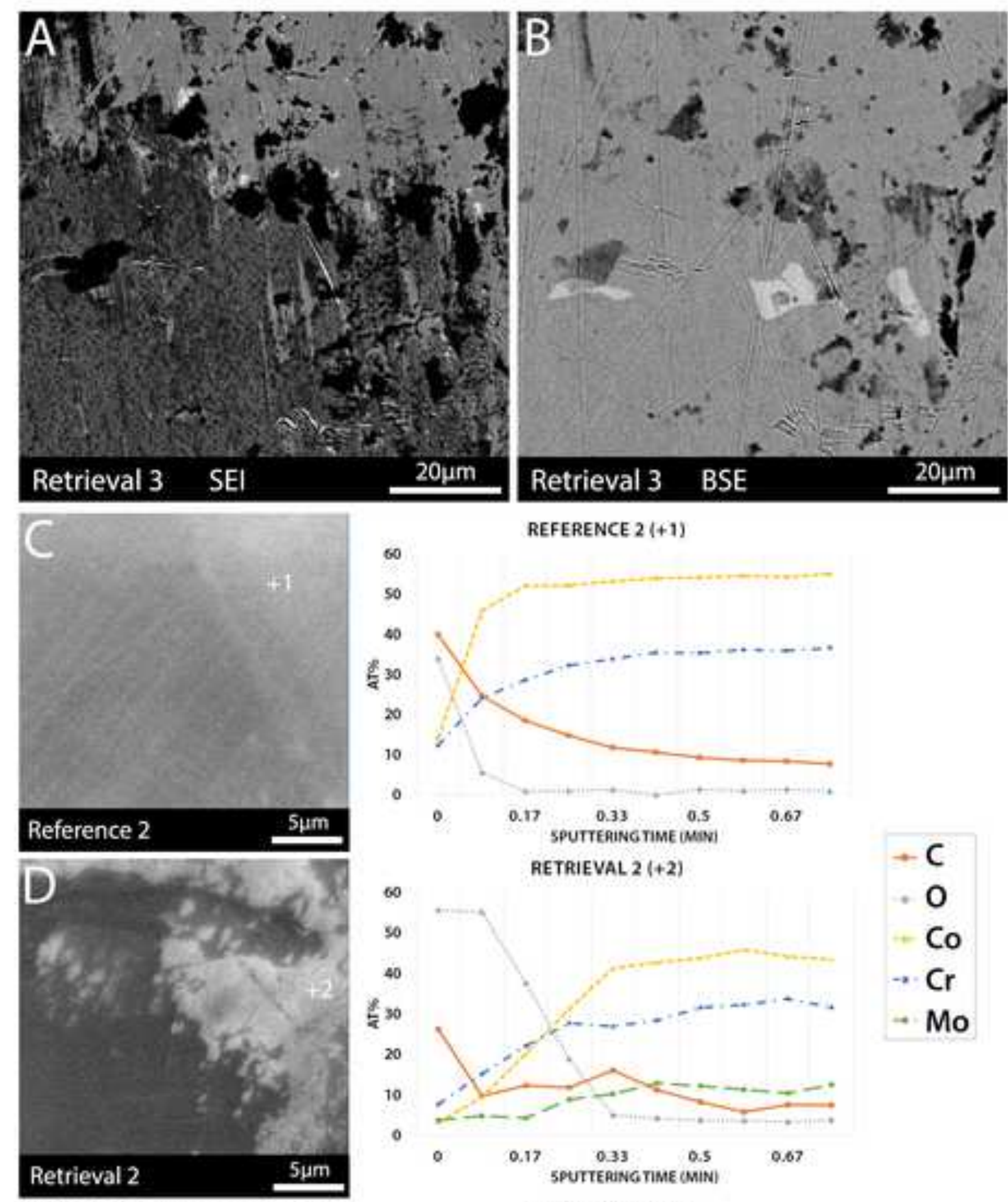

REFERENCE $2(+1)$
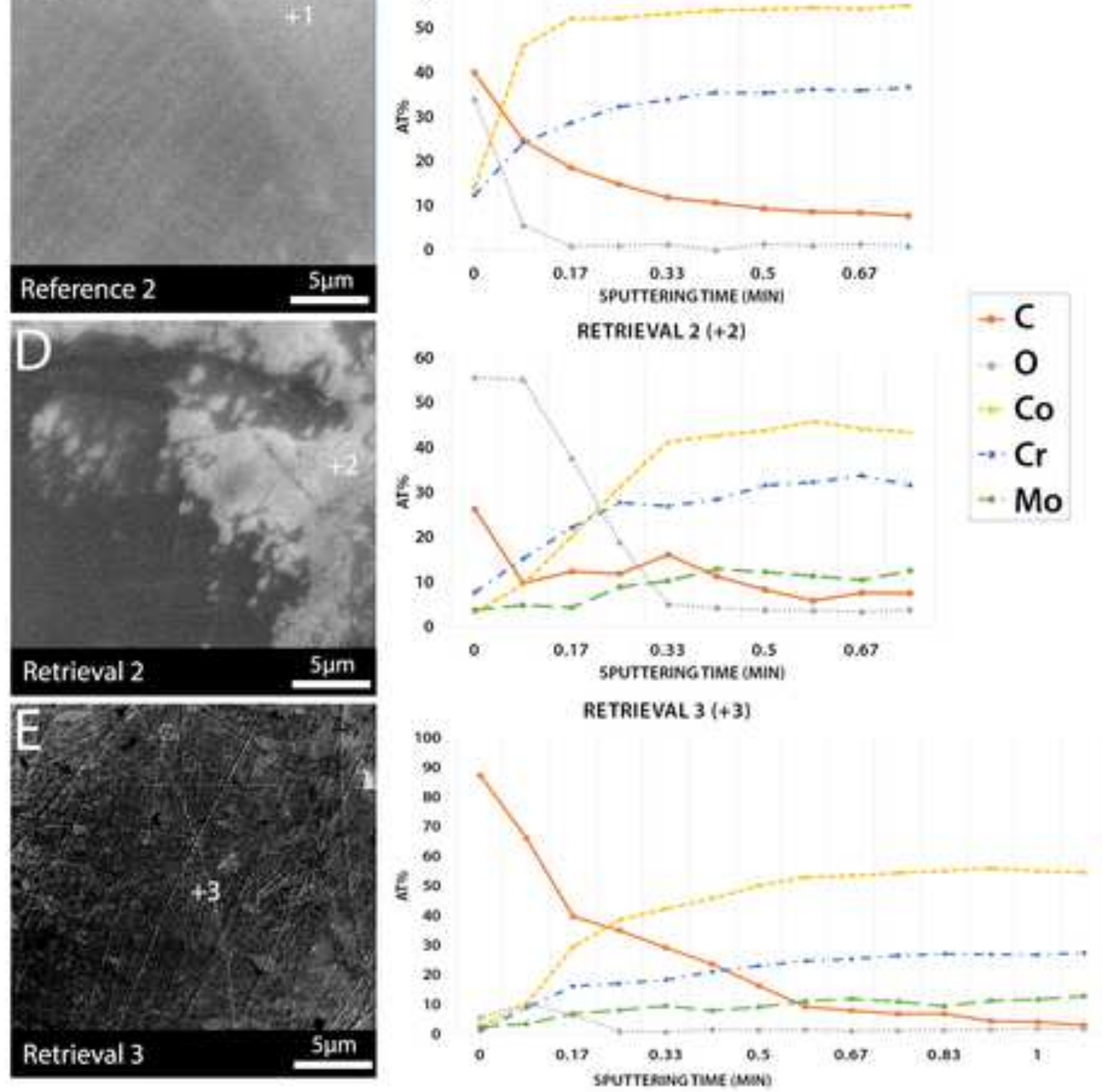

Figure 4 

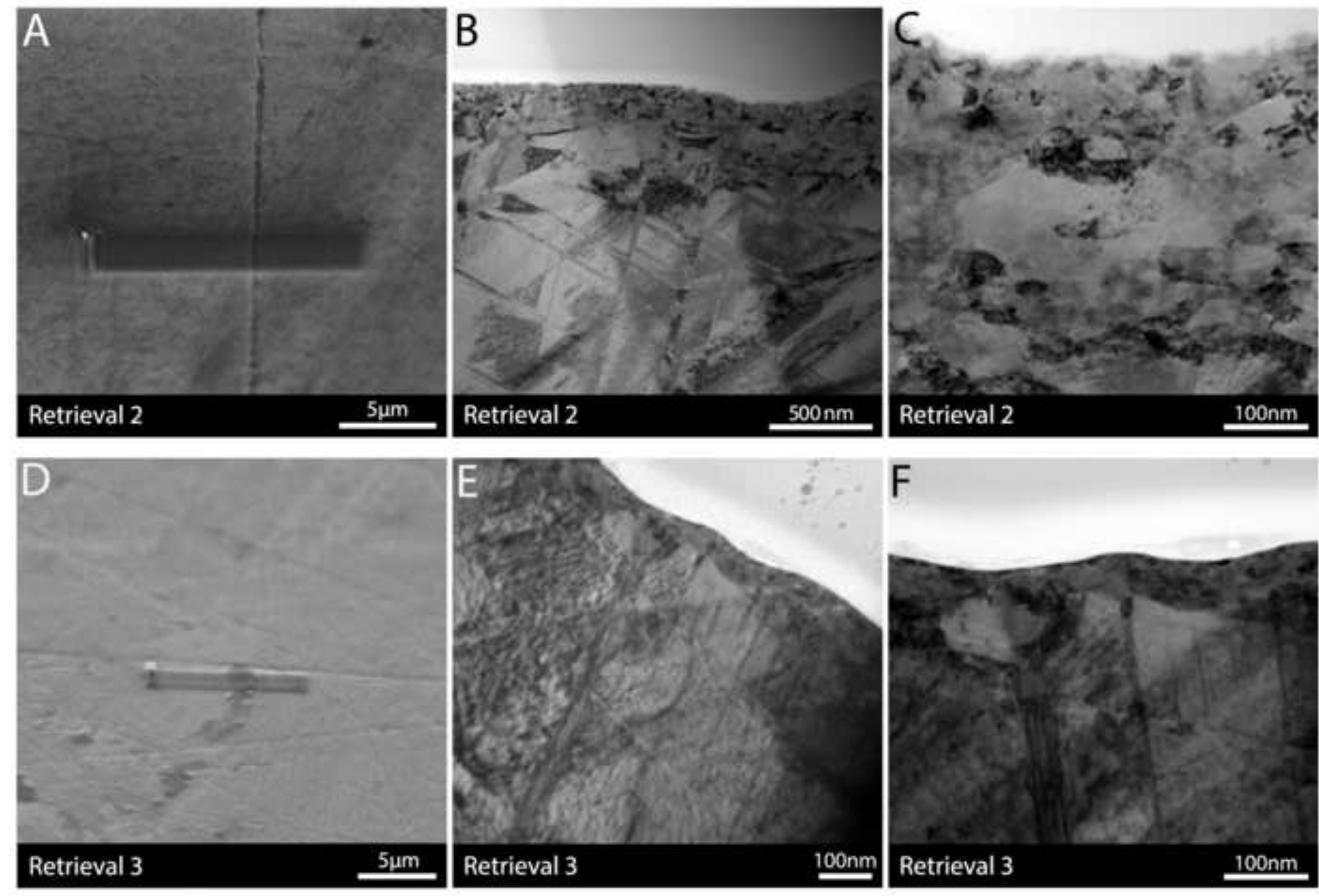

F

Figure 5 
A
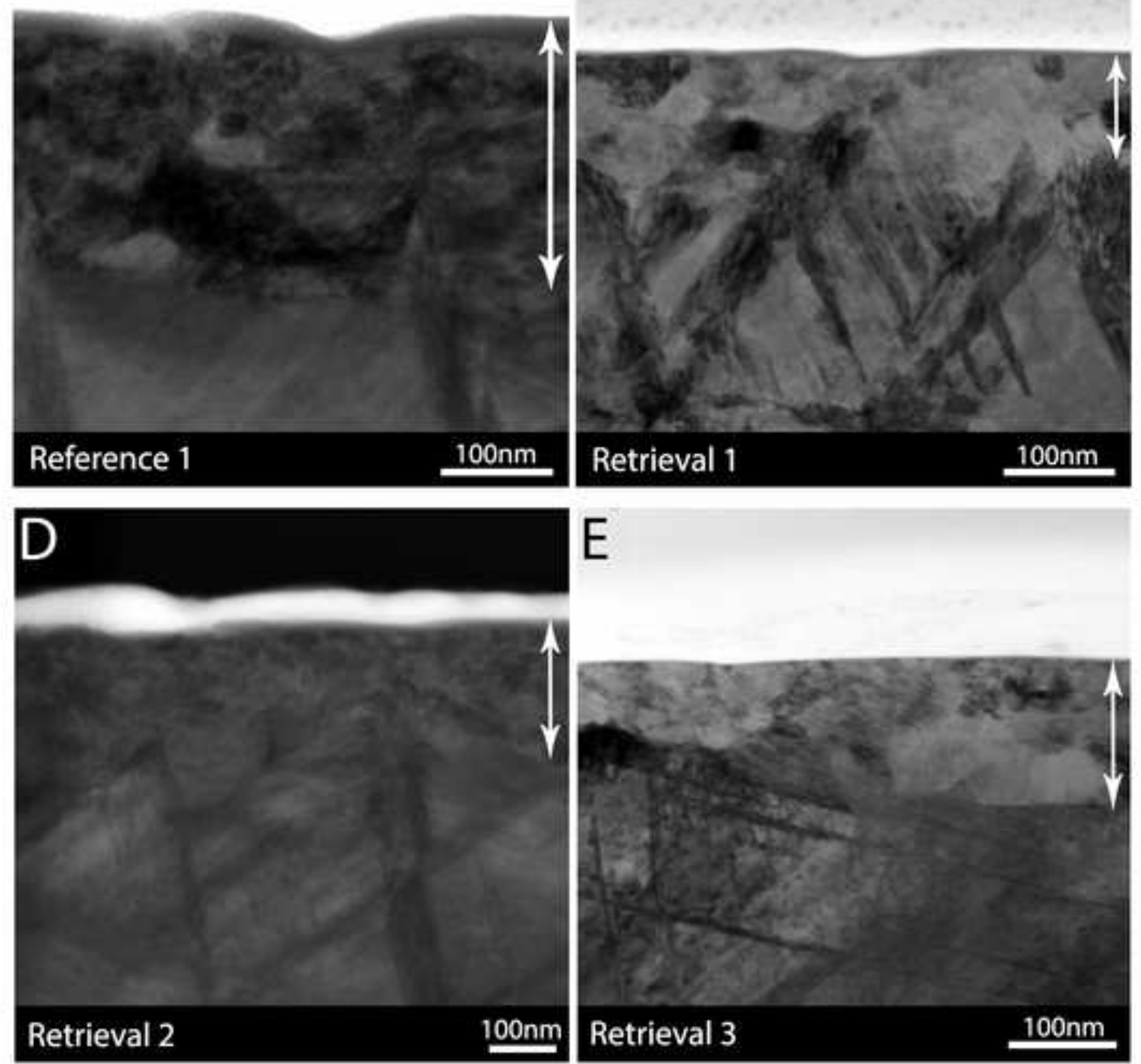

Figure 6 


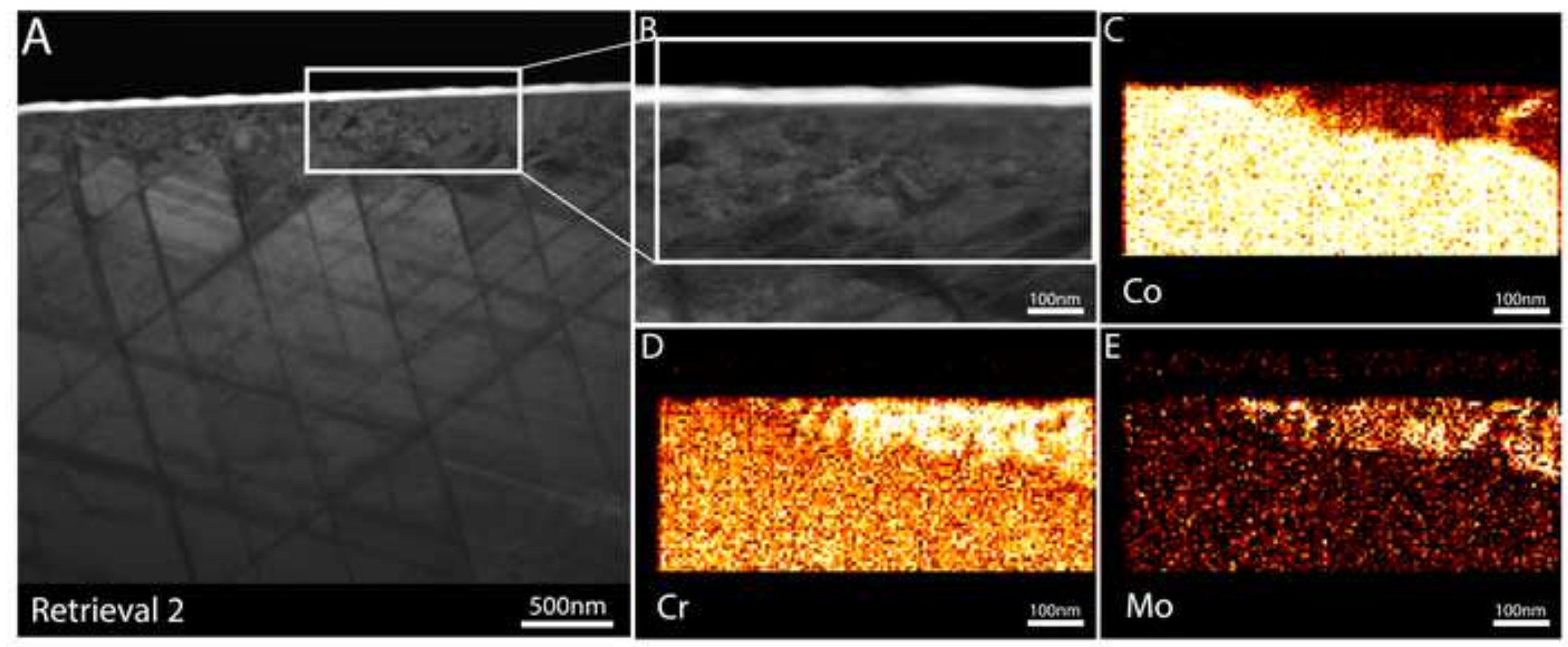

Figure 7 